\title{
Correlations between Individual Features of Electrodermal Response During Viewing of the Same Videos
}

\author{
Vladimir Ivanovich Mirgorodsky ${ }^{1}$, Valery Vasilievich Dementienko ${ }^{1}$, Vadim Vladimirovich Gerasimov ${ }^{1}$, \\ Sergei Vladimirovich Peshin ${ }^{1}$,Vladimir Borisovich Dorokhov ${ }^{2}$, Viacheslav Markovich Shakhnarovich ${ }^{3}$ \\ ${ }^{1}$ Fryazino branch of the Institution of Russian academy of sciences Kotel'nikov Institute of Radio Engineering and Electronics of RAS \\ ${ }^{2}$ Institute of Higher Nervous Activity and Neurophysiology, Russian Academy of Sciences, Moscow \\ ${ }^{3}$ CJSC NEUROCOM, Moscow \\ *Corresponding Author: vim510488@gmail.com
}

Copyright (C) 2013 Horizon Research Publishing All rights reserved.

\begin{abstract}
In the past few years, much attention has been given to the development of neurophysiological methods of analyzing social interactions between subjects in response to natural stimuli presented in videos. This article describes a methodological approach for assessing similarities, and differences in how videos are perceived by different subjects based on their phasic electrodermal response (EDR). Experiments with viewing of videos revealed statistically significant correlations of electrodermal reactions in different subjects that made it possible to distinguish groups with similar or differing types of perception of the films. The results obtained demonstrate to our mind the possibility of using EDR as a simple method for quantifying autonomous nervous activity to assess the emotional, and activation components of interpersonal interaction.
\end{abstract}

Keywords Electrodermal Activity, Phasic Electrodermal Reaction, Individual Differences, Correlation of Simultaneous Perception

\section{Introduction}

Researchers are increasingly attracted to studies of collective activities that use unbiased methods [1-5].

The transition to research of the neurobiological basis of social interaction made it necessary to bring experimental conditions closer to reality, and to study the physiological reactions of two or more directly interacting individuals. The analysis of dynamic aspects of social interaction has required the development of methods of simultaneous recording of physiological characteristics of two or more individuals. Existing techniques are capable of analyzing brain structure activities by neurovisualization methods, such as functional magnetic resonance imaging applied simultaneously to two individuals separated by a certain distance who communicate via the Internet [6]. However, this informative method is relatively inertial: time resolution approximates to one second or even higher. Electroencephalogram recording allows for studying faster brain processes during joint, activities performed by two [3, 7, 8] and even four individuals $[9,10]$. The complexity of simultaneous recording of brain structure activity using an functional magnetic resonance imaging scanner necessitated the development of "robust inter-subject correlation of hemodynamic brain activity when subjects freely viewed a video clip" [2,8,11-16].

Recording autonomic indices such as heart rate, respiration rate, blinking, and electrodermal activity allows us to assess non-specific features of social interaction associated with emotional, and activation components [14]. Recording these indices is more feasible than tomography, and electroencephalography, but we did not find any studies that recorded these indices during joint activities. These methods were only used to study inter-individual variations during the repeated presentation of socially meaningful stimuli [13, 14].

Electrodermal activity (EDA) is the variation of skin electrical properties $[17,18]$. It is one the best-known electrophysiological parameters and is widely used in psychophysiological and psychological studies. There are tonic EDA changes with a constant time period of several minutes, phasic changes EDR with a constant time period of several seconds that are closely related to the orientation reflex [19], and EDR without any registered stimuli (non-specific or spontaneous).

Recording electrodermal EDA provides a number of advantages compared to other indicators of autonomic nervous system activation. First, the reliable extraction of the EDR signal in response to a single stimulus does not require many summations, such as in psychophysiological studies of 
rare situations, or the occurrence of meaningful stimuli of endogenous origin [20]. Second, it was proven that EDR occurrence is mostly related to informational, emotional, and other non-somatic components of body activation [18]. Finally, the eccrine sweat glands that determine EDR genesis are known to have purely sympathetic innervation, unlike other organs that are controlled by combined sympathetic and parasympathetic innervations [18].

In our earlier studies [21, 22], EDR recording methods were applied to analyze a decrease in wakefulness during monotonous activity in repeated experiments, as well as to study gender differences [23]. To identify the moment of EDR appearance, we applied signal detection algorithms that we developed based on EDR signal shape [21].

Our study was the continuation of [24] and was aimed at assessing the efficiency of phasic EDR for analyzing paired activity in terms of perception of the same videos (in the available sources we found no studies involving comparison of EDRs of two persons). The objective was to assess EDR efficiency in identifying perception variability between individuals in the course of the same video watching. We analyzed EDR signal correlation of all participants who viewed the same video. For comparative assessment of inter-individual variability, the intra-individual variability of two subjects in the course of repeated viewing of the same videos was analyzed.

\section{Methods}

\subsection{Stimulus}

Clips from two feature films were used as stimuli: "Transporter 3", 2008, Lionsgate, part one, 1 hour long (Film 1), and "The Lives of Others", 2006, Sony Pictures Classics, two parts, 1.5 hours each (Films 2, and 3).

\subsection{Procedure}

Nineteen individuals were tested, including 10 males, and 9 females, aged 21 - 75. Usually experiments were conducted with two subjects; but a few experiments were conducted with one subject. The experiments were performed in an isolated room, with daylight, and a comfortable ambient temperature ranging from 20 to $25^{\circ} \mathrm{C}$. The subjects were asked to remain in a quiet state to prevent any artifact signals related to movement. The subjects were seated on chairs viewed a film on a 17-22" monitor. EDR from subjects was recorded to computer.

EDA signals were recorded from the middle finger of the left hand. A total of 11 recordings were performed with the film "Transporter-3," and 10 recordings were made with "The Lives of Others" parts 1, and 2. The subjects were instructed to watch the films without talking, and with as little motion as possible. A description of the subjects and their distribution by film is shown in Appendix 1. As a rule, the subjects were not familiar with the film they were going to watch during experiments. A maximum of one experiment was performed per day.

The study was performed in accordance with the guidelines of the Helsinki declaration, and written voluntary consent was obtained from each subject prior to their participation in the study.

\subsection{Recording}

EDA recordings were performed according to the Fere method [28], i.e., we applied two electrodes to a finger to measure skin resistance in response to current below $10 \mu \mathrm{A}$. Semi-circular copper electrodes were used to cover the inner part of the third phalanx of the finger. The width of the electrodes was $6 \mathrm{~mm}$, and the distance between them was 5 $\mathrm{mm}$. The "dry method" was applied, with no electrode paste. The recording equipment was selected based on the 24-digit sigma-delta analog-digital converter (NEUROCOM, Moscow). This equipment is capable of simultaneously recording EDA signals via two independent channels, with a sampling rate of $8.2 \mathrm{~Hz}$. Efficient filtration of $\mathrm{AC}$ interference noise at $50 \mathrm{~Hz}$ made it possible to record the EDA with high precision: the mean square error of resistance measurements normally did not exceed $10 \mathrm{Ohm}$. For a mean measured interelectrode resistance of approximately 100 $\mathrm{kOhm}$ the relative error is $0.01 \%$, which was quite sufficient for reliable recording of EDR signals with relative values of over $0.2 \%$ across the whole resistance range used. Interelectrode resistance in laboratory conditions (at approximately $20^{\circ} \mathrm{C}$ ) normally ranges from $10 \mathrm{kOhm}$ to $1 \mathrm{MOhm}$. In the course of the experiment, EDA signals were simultaneously recorded to the computer hard drive via two channels.

\subsection{Data Processing}

We carried out data processing for calculating the correlation between EDR signals recorded in different experiments in three stages. Under correlation processing were used EDR signals obtained in joint viewing and in viewing in different experiments.

During the first stage, the EDR signal positions were determined from the EDA recordings. A purpose-specific algorithm [23]was applied, making it possible to detect EDR signals with relative values exceeding $0.2 \%$.

Appendix 2 shows the operating principle of the algorithm for determining EDR signal positions from the EDA signals. The algorithm gives the rows of values of time positions of registered EDR signals.

In the second stage, we performed correlations on the signals, which yielded normalized correlation values between EDR signal positions obtained from different experiments. Correlation details are shown in Appendix 3. This step resulted in normalized correlation coefficients for each pair of EDA signal recordings obtained from the experiments. 
In the third stage, we assessed the probability of accidental value correlation obtained during the second stage of data processing. This step was necessary because it was unclear if the correlation values of EDR signals obtained were random or actual. In other words, a validity appraisal of the result was performed. Estimation of accidental occurrences of the obtained correlation coefficients was performed using two methods: mathematical (computer) modeling, and analytical calculation. Details on the estimation of accidental occurrences of correlation rates are shown in Appendix 4.

\section{Results}

Below are the diagrams of EDA signals of two subjects in the course of viewing "Transporter 3."

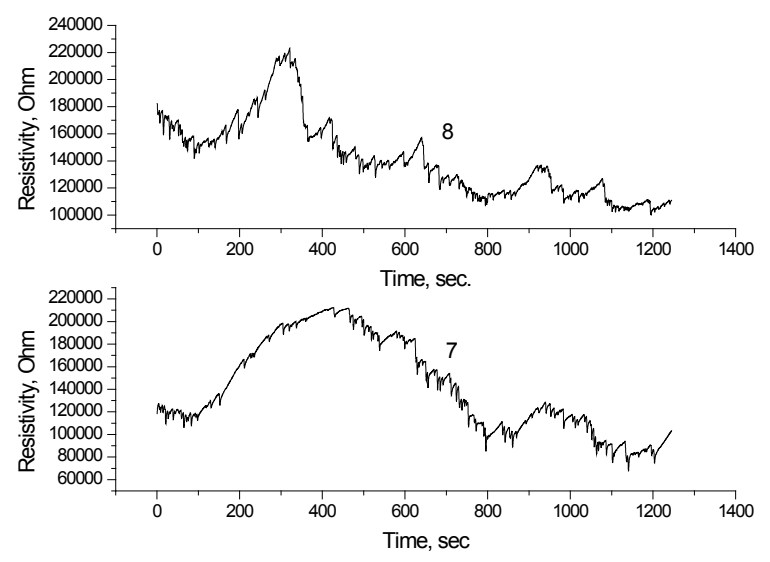

Figure 1. Simultaneously obtained skin resistivity plotted against time in subjects 7 , and 8 . Electrodes were located on the middle fingers of the left hand.

In this case, we observed mostly clear EDA signals without interference. In this case, correlations between EDR signals are almost not visible. However, they can be better appreciated in the enlarged fragment shown in Figure 2, which is an example of extracting EDR from the EDA recording using the algorithm described above.

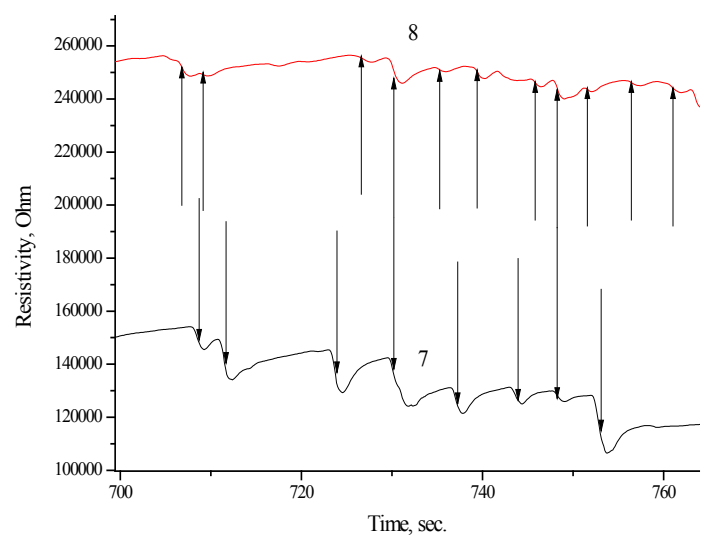

Figure 2. Fragments of signals showed in Fig. 1. The arrows show the positions of EDR signals. Two cases of EDR coincidence are visible.
Both graphs show signals that have the pattern specific for EDA. However, the EDR signal occurrences on the different graphs (with different subjects) typically do not coincide, as may be noted from the arrows showing the positions of EDR signals on a horizontal timeline. Only 2 coinciding occurrences of EDR signals from two different subjects are recorded marked with continuous arrows between the two graphs.

As mentioned before, normalized correlation coefficients, and probabilities of accidental correlation coefficient occurrence were calculated for each pair of EDR signal position rows. We performed further data processing by analyzing these values.

While the number of subject pairs with data that has undergone correlation analysis may be large (55 for "Transporter 3"), the tables below show only the highest values of EDR signal correlation coefficient along with their accidental occurrence probabilities arranged in descending order. The tables contain the data with accidental occurrence probabilities of less than or equal to $5 \%$, i.e., the most reliable data.

The results obtained during different films need to be considered individually.

Table 1. Shows the obtained results from "Transporter 3"

\begin{tabular}{|c|c|c|c|c|}
\hline \multirow{2}{*}{$\begin{array}{c}\text { Subject 1 } \\
\text { Code }\end{array}$} & \multirow{2}{*}{$\begin{array}{c}\text { Subject 2 } \\
\text { Code }\end{array}$} & Correlation, \% & \multicolumn{2}{|c|}{$\begin{array}{c}\text { Probability of accidental } \\
\text { occurrence }\end{array}$} \\
\cline { 4 - 5 } & 2 & 17.9 & Simulation & Calculation \\
\hline 2 & 4 & 17.3 & 0.1 & 0.1 \\
\hline 11 & 2 & 16.5 & 0.1 & 0.0 \\
\hline 11 & 8 & 15.7 & 0.4 & 0.1 \\
\hline 2 & 8 & 15.5 & 0.4 & 2.2 \\
\hline 2 & & &
\end{tabular}

Table 1.The first column lists the codes of the first subject; the second column lists the codes of the second subject in a pair; the third column shows the normalized correlation coefficients of the EDR signals of these subjects; and the fourth, and the fifth columns show the probabilities of accidental occurrence of the correlation coefficients obtained: simulated (4), and calculated (5).

The maximum value of correlation for different experiments corresponds to element $(2,2)$, and has a correlation value of almost $18 \%$, with a probability of accidental occurrence as low as $0.1 \%$. This element was obtained in a single subject who watched the same film in different experiments. The additional elements are as follows: $(11,4)$ with a correlation of $17.3 \%$, and a probability of accidental occurrence below $0.1 \% ;(11,2)$ with a correlation of $16.5 \%$, and a probability of accidental occurrence $0.1 \%$; $(2,8)$ with a correlation of $15.7 \%$, and a probability of $0.4 \%$; and $(2,8)$, which was a different experiment with the same subjects, with a correlation of $15.5 \%$, and an accidental occurrence probability of $0.4 \%$.

The probabilities of accidental occurrences are sufficiently low, and confirm the statistical significance of the results.

The results indicate that EDR signal correlation value is 
not directly related to the probability of accidental occurrence due to the reasons explained below.

Therefore, we can identify the pairs of subjects whose signals are correlated with each other: $(2,2),(11,4),(11,2)$, and $(2,8)$. Here, we only have pair correlations, but on this basis we can obtain information on correlations within larger groups of subjects because some of the subjects are found in the table of significant pair correlations several times. For example, one can find this for subject 2 three times, and twice for subject 11 . Because subjects 2 and 11 were in pairs with each other as well as with other subjects, their figures are also likely to incorporate other mentioned cases. Specifically, we can suppose that perception of the material by the subjects $2,11,4$, and 8 has similar features.

Table 2. shows the obtained results from Part 1 of "The Lives of Others".

\begin{tabular}{|c|c|c|c|c|}
\hline & & & \multicolumn{2}{|c|}{$\begin{array}{c}\text { Probability of accidental } \\
\text { occurrence }\end{array}$} \\
\cline { 4 - 5 } $\begin{array}{c}\text { Subject } \\
\text { 1 Code }\end{array}$ & $\begin{array}{c}\text { Subject } \\
\text { 2 Code }\end{array}$ & Correlation, \% & Simulation & Calculation \\
\hline 14 & 15 & 13.3 & 0.0 & 0.0 \\
\hline 15 & 17 & 12.8 & 0.2 & 0.2 \\
\hline 4 & 8 & 12.7 & 0.8 & 0.7 \\
\hline 17 & 4 & 12.0 & 3.8 & 5.5 \\
\hline 14 & 4 & 11.8 & 1.0 & 1.6 \\
\hline
\end{tabular}

Table 2 - The first column lists the codes of the first subject; the second column lists the codes of the second subject in a pair; the third column shows the normalized correlation coefficients of the EDR signals of these subjects; and the fourth, and the fifth columns show the probabilities of accidental occurrence of the correlation coefficients obtained: simulated (4), and calculated (5)

Analysis of the table's elements shows that the maximum value of correlation for different subjects corresponds to element $(14,15)$ with $13.3 \%$, and a probability of accidental occurrence below $0.1 \%$. Next is element $(15,17)$, with a correlation of $12.8 \%$, and an accidental occurrence probability of $0.2 \%$; element $(4,8)$ with a correlation value of $12.7 \%$, and an accidental occurrence probability of $0.8 \%$; followed by $(17,4)$ with a correlation of $12 \%$, and a probability of $3.8-5.5 \%$; and element $(14,4)$ with a correlation of $11.8 \%$, and a probability of $1-1.6 \%$.

The results listed in Table 2 indicate that the degree of EDR signal coincidence is not directly related to the probability of accidental occurrence due to the reasons explained below.

The analyzed data allows us to distinguish subjects whose EDR signals correlate with each other. First, for pairs (14, $15),(15,17),(4,8),(17,4)$, and $(14,4)$, we only have pair correlations, but on this basis we can obtain information on correlations within larger groups of subjects because some of the subjects are found in the table of significant pair correlations several times. For example, subject 4 occurred in different combinations three times, subjects 14,15 , and 17 occurred in different combinations twice. The results of subject 4 coincide with those of subjects $8,14,15$, and allow us to speak of common features of perception of the presented material in subjects $4,8,14,15$, and 17 .
Analysis of the table's elements shows that the maximum degree of signal correlation for different subjects corresponds to the element $(10,11)$, and equals $12.6 \%$, with the probability of accidental occurrence at $0.1 \%$. Next comes element $(2,4)$, with a correlation of $12.3 \%$, and an accidental occurrence probability of $2.8 \%$; element $(17,10)$, with a correlation of $12.3 \%$, and an accidental occurrence probability of $0.2 \%$; element $(14,15)$, with a correlation of $11.8 \%$, and an accidental occurrence probability of below $0.1 \%$; and element $(16,17)$, with a correlation of $10.7 \%$, and an accidental occurrence probability of $1.2 \%$.

Table 3. shows the obtained results from Part 2 of "The Lives of Others".

\begin{tabular}{|c|c|c|c|c|}
\hline \multirow{2}{*}{$\begin{array}{c}\text { Subject 1 } \\
\text { Code }\end{array}$} & \multirow{2}{*}{$\begin{array}{c}\text { Subject 2 } \\
\text { Code }\end{array}$} & Correlation, \% & \multicolumn{2}{|c|}{$\begin{array}{c}\text { Probability of accidental } \\
\text { occurrence }\end{array}$} \\
\cline { 4 - 5 } & & Simulation & Calculation \\
\hline 10 & 11 & 12.6 & 0.1 & 0.0 \\
\hline 2 & 4 & 12.3 & 2.8 & 2.9 \\
\hline 17 & 10 & 12.3 & 0.2 & 0.4 \\
\hline 14 & 15 & 11.8 & 0.0 & 0.0 \\
\hline 16 & 17 & 10.7 & 1.2 & 1.8 \\
\hline
\end{tabular}

Table 3 - The first column lists the codes of the first subject; the second column lists the codes of the second subject in a pair; the third column shows the normalized correlation coefficients of the EDR signals of these subjects; and the fourth, and the fifth columns show the probabilities of accidental occurrence of the correlation coefficients obtained: simulated (4), and calculated (5).

Again, the results indicate that the degree of EDR signal coincidence is not directly related to the probability of accidental occurrence due to the reasons explained below.

Our interpretation of the above elements enabled us to single out subjects with correlating values: $(10,11),(2,4)$, $(17,10),(14,15)$, and $(16,17)$. In this case, it is also possible to extrapolate the results of calculating paired correlations to the analysis of larger groups of subjects. Again, some subjects are on the list of significant paired correlations more than once. Note subjects 10 , and 17 , which occur twice in different combinations. The results of subject 10 coincide with those of subjects 11,17 , and the results of subject 17 coincide with 10 , and 16 , which allows us to suggest similar features of perception of the presented materials by the group including these four subjects. Conversely, subjects 14, 15, 2, and 4 demonstrate no common significantcorrelations (within a group of more than two subjects).

\section{Discussion}

The results suggest the possibility of quantitative estimation of individual subject responses in the course of perceiving similar video materials based on phasic EDRs. In most cases, similar reactions were detected in subjects of different pairs, which mean that the similarity of reactions is mostly based on individual response to the storyline rather than simultaneous watching of the film. It may be supposed that in the course of simultaneous activities, the correlation 
coefficients of physical EDR indicators would be higher, similar to that demonstrated for EEG in during the joint performance of music (Babiloni et. al., 2010, 2011).

The highest correlation value $(17.9 \%)$ was recorded in subject 2, who viewed Film 1 more than once. However, this does not mean that repeatedly viewing a film results in especially high correlation coefficients. The lack of sufficient statistical data does not allow for such a conclusion; moreover, as pertains to other films, the maximum correlation coefficients were registered with different subjects. In particular, in the course of repeated viewing of "The Lives of Others" by subject 11 , the correlation $(8.7 \%)$ was far below the maximum value for this film $(12.6 \%)$ obtained by the pair $(10,11)$.

The probabilities of accidental occurrences in Tables 1-3 obtained by simulation, and calculations are similar in magnitude, with the accuracy up to statistical uncertainties. Discrepancies of these values are, from our point of view, related to effects of EDR signal position uniformity on the probability of accidental occurrence distribution in the course of simulation. Considering the equal probability of signal occurrence within the whole duration of experiment, the less uniformly signals are distributed, the lower is the probability of accidental occurrence of such a distribution.

It should also be noted that using EDA signals for evaluating how socially significant information is perceived may turn out to be highly promising compared with more technically complicated methods. This is due to the availability of techniques for obtaining EDA signals from a relatively large number of subjects (100, and more), even if they are far away from each other. We believe that applying this method may advance the development of social studies.

The work described here allows us to conclude that the method may be useful for assessing patterns of social interaction between individuals [25-27]. Further studies will be focused on this point.

\section{Conclusions}

1. The method of EDR signal position detection, and the procedure developed for correlation analysis allowed us to calculate the correlations between EDR signals obtained from different subjects with a sufficiently high degree of reliability, as determined by calculations, and computer simulations.

2. The possible availability of correlating EDR signals obtained from several subjects, which viewed the same video materials was demonstrated for the first time. The degree of correlation of such signals normally did not exceed $20 \%$, while the probability of accidental occurrence of coincidence of the EDR signals was low, averaging below $3-5 \%$.

3. Analysis of the mutual correlation of EDR signals makes it possible to our mind to single out certain subject groups displaying a similar type of perception information, which may be useful, for example, when selecting partners for activities.

\section{Appendix 1}

\begin{tabular}{|c|c|c|c|c|c|}
\hline \multicolumn{3}{|c|}{ Subject parameters } & \multicolumn{3}{|c|}{ Quantity of experiments } \\
\hline $\begin{array}{l}\text { Subject } \\
\text { code }\end{array}$ & Sex & $\begin{array}{l}\text { Age, } \\
\text { years }\end{array}$ & $\begin{array}{c}\text { Transporter, } \\
\text { part } 3\end{array}$ & $\begin{array}{c}\text { The } \\
\text { Lives of } \\
\text { Others, } \\
\text { part } 1\end{array}$ & $\begin{array}{c}\text { The } \\
\text { Lives of } \\
\text { Others, } \\
\text { part } 2\end{array}$ \\
\hline 1 & Female & 75 & 1 & 0 & 0 \\
\hline 2 & Female & 26 & 2 & 0 & 1 \\
\hline 3 & Male & 36 & 1 & 0 & 0 \\
\hline 4 & Male & 24 & 1 & 1 & 1 \\
\hline 5 & Female & 25 & 1 & 0 & 0 \\
\hline 6 & Female & 24 & 1 & 0 & 0 \\
\hline 7 & Female & 26 & 1 & 0 & 0 \\
\hline 8 & Female & 26 & 1 & 1 & 0 \\
\hline 9 & Male & 62 & 0 & 1 & 0 \\
\hline 10 & Male & 45 & 1 & 0 & 1 \\
\hline 11 & Male & 40 & 1 & 2 & 1 \\
\hline 12 & Male & 38 & 0 & 1 & 0 \\
\hline 13 & Male & 26 & 0 & 1 & 0 \\
\hline 14 & Female & 21 & 0 & 1 & 1 \\
\hline 15 & Female & 28 & 0 & 1 & 1 \\
\hline 16 & Male & 45 & 0 & 0 & 1 \\
\hline 17 & Male & 43 & 0 & 1 & 1 \\
\hline 18 & Female & 45 & 0 & 0 & 1 \\
\hline 19 & Male & 25 & 0 & 0 & 1 \\
\hline
\end{tabular}

\section{Appendix 2}

Determination of EDR signal positions from EDA signal recordings

Fig. 1 shows an example of the algorithm for determining EDR position from the EDA recording.

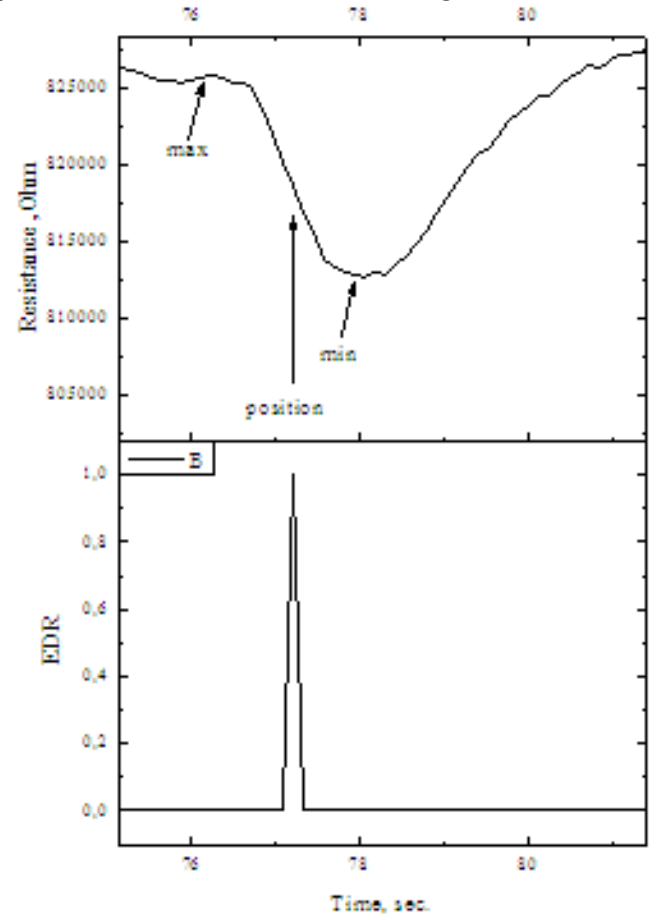

Figure 1. Illustration of the algorithm action. Top graph: EDA signal, bottom graph: EDR signal position, which was detected by the algorithm 
As the graphs illustrate, EDR occurrence is considered as the moment between the maximum point in the beginning of EDR (marked as max.), and minimum point (marked as min.), with the accuracy defined by a signal sampling rate of approximately $8.2 \mathrm{~Hz}$. Thus, at the first stage of processing, EDA signals obtained in the course of the experiment as interelectrode resistivity against time were converted into data on EDR signal positions along the timeline represented by 0 , where the EDR was not detected, and 1 , where it was detected.

\section{Appendix 3}

Algorithm for correlating EDR signal positions.

The comparison included the data from all subjects, who had seen the same film. Operations employed in the course of correlation processing are illustrated in Fig. 2

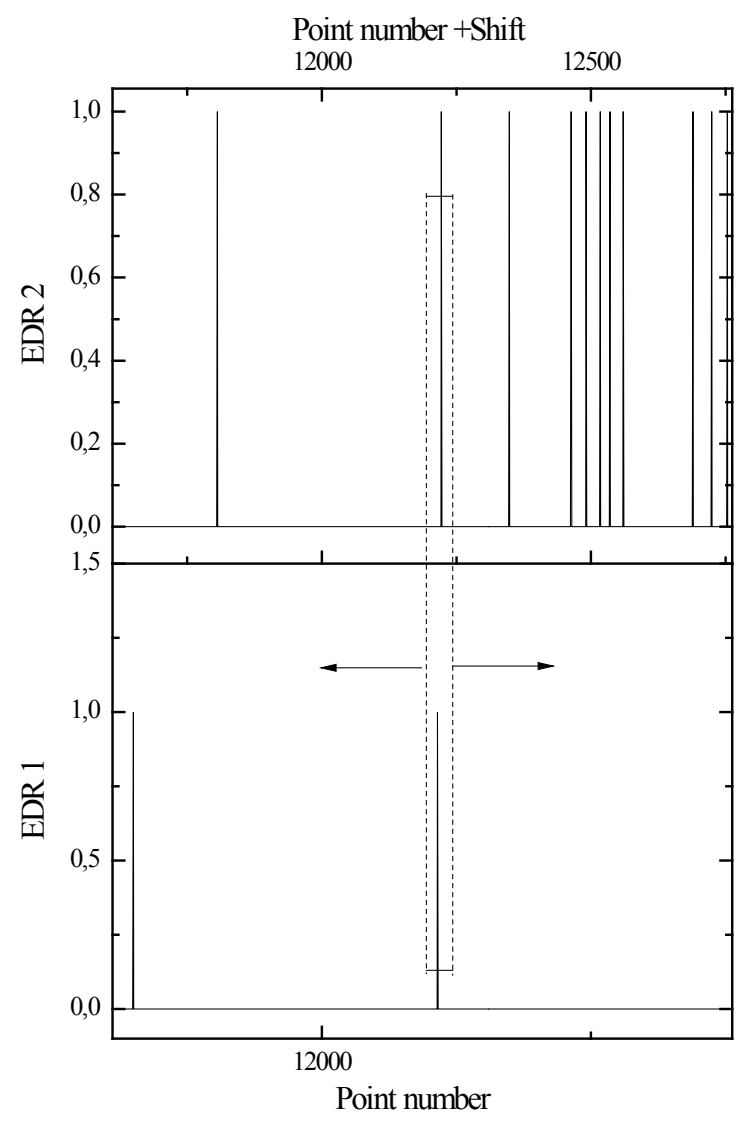

Figure 2. Operations in the course of correlation analysis.

Fig. 2 (top and bottom) shows two series of positions of EDR signals from different experiments versus time. Despite the fact that experimental data were obtained while the same films were presented, the moment that the recording started could have varied slightly in different experiments due to technical reasons. Therefore, correlating was performed with a variable shift of EDR positions rows relative to each other within the range of $\pm 20 \mathrm{sec}$. In the process of correlation analysis, for each value of the shift of EDR signal position relative to each other, we counted the number of cases where EDR signal positions differed by less than \pm 0.3 seconds. An example of this is shown in fig. 2 by the dashed line. The result obtained represented the maximum number of EDR signals. The resulting number was divided by the geometric mean of numbers of the EDR signals in both channels, and the result was expressed as a percent. The value obtained represents the normalized correlation coefficient between the positions of EDR signals in both channels. For example, the correlation coefficient for two identical signals is $100 \%$. The correlation coefficient for two completely different signals is close to $0 \%$

\section{Appendix 4}

Defining the probability of accidental occurrence of correlation coefficients between EDR position rows

Mathematical modeling was performed after the correlation coefficient "K" of two sequences of EDR signal positions (S1, and S2) was obtained at the second stage of data processing. For this purpose, correlation analysis was performed similar to that described above but with the other sequences of EDR positions signals. The first was the initial $\mathrm{S} 1$, obtained from experimental data, and the other was a computer-generated sequence with the length, and number of EDR in S2 but with a random signal distribution. Positions were obtained using a standard algorithm of random signals with uniform distribution. An random, uniform distribution of EDR signals along the experimental timeline may be justified by experimental evidence - a random distribution of EDR signals along the timeline is normally observed in the course of the experiments without strong stimulations. As a rule, 12,000 computer simulations were performed. At the same time, the number of experiments was determined in which correlation coefficients between the signals were equal to or higher than those " $K$ " obtained from the experimental data. After that, the resulting sum was divided by the total number of simulations $(12,000)$ to obtain the normalized value representing the probability of accidental occurrence of the correlation coefficient obtained from the experimental data. To estimate the accuracy of such calculations, 120,000 computer-simulated experiments were performed, and these simulations yielded the same values of the probability estimates as the 12,000 simulations (within the accuracy of statistical error).

It should also be noted that the above described computer modeling yielded correlation coefficients with Gaussian distribution. The highest correlation values from Tables 1-3 exceed the mean square deviation of this distribution by 2-3 folds. This means that the probability of accidental occurrence of obtained correlation values were quite low (0.3 - 5\%).

Another method of evaluating the probability of accidental occurrence of experimental results is a calculation that permits obtaining the said probability based on combinatory 
analysis from the following formula:

$$
P=\frac{\left(N-K_{2}\right) ! * K_{2} ! *\left(N P^{*} K_{1}\right) ! *\left(N-N P^{*} K_{1}\right) !}{N ! *\left(N P^{*} K_{1}-C\right) !\left(K_{2}-C\right) ! *\left(N-N P^{*} K_{1}-K_{2}+C\right) !}
$$

where $\mathrm{N}$ is the number of counts, $\mathrm{K} 1$, and $\mathrm{K} 2$ are the numbers of the EDR signals detected, $\mathrm{C}$ is the number of coincident EDR signals in the sequences being compared, and NP is the number of counts determining the interval within which EDR signals are considered to be coincident. Typical values of formula elements (such as during experiments) are as follows: $\mathrm{N} \approx 32,000, \mathrm{~K} 1, \mathrm{~K} 2 \approx 300, \mathrm{C} \approx 60$, $\mathrm{NP}=7$.

The formula is based on counting the number of various realizations of random EDR signal positions. First, the number of possible placements of "C" EDR signals along "K2*NP" possible positions was calculated, taking into account the "NP" interval, within which EDR signals are considered to be coincident.

Next, the number of placements of non-coinciding "K2-C" EDR signals on "N-NP*K1" locations was calculated.

The product of these values represents the number of possible realizations of coincidence of " $\mathrm{C}$ " EDR signals. This number was then divided by the total number of possible placements of "K2" EDR signals on "N" possible positions, which allowed us to obtain the probability value by formula (1).

\section{REFERENCES}

[1] Social Interaction: From Concepts to Brain Im4ing. // Physiol. Rev. 2009. V.89.P.453-479.

[2] Hasson U., Nir Y., Levy I., Fuhrmann G., Malach R.Intersubject synchronization of cortical activity during natural vision. // Science, 2004. V.303 (5664).P.1634-1640.

[3] Wackermann J. Dyadic Correlations between Brain Functional States: Present Facts and FuturePerspectives. // Mind and Matter. 2004. V.2. N.1.P.105-122

[4] Frith C, Frith U. Interacting minds: a biological basis. // Science.1999. V.286.P.1692-1695.

[5] Chadi C., Andreasen N.C., O’Leary D. S. Visualizing How One Brain Understands Another: A PET Study of Theory of Mind. // Am. J. Psychiatry. 2003. V.160, P.1954-1964.

[6] Montague P.R., Berns G.S., Cohen J.D., McClure S.M., Pagnoni G., Dhamala M., Wiest M.C., Karpov I., King R.D., Apple N., Fisher R.E. Hyperscanning: simultaneous fMRI during linked social interactions. // Neuroimage. 2002. V.16. P.1159-1164.

[7] Tognoli E., Lagarde J., DeGuzman G.C., Kelso J.A. The phi co14lex as a neuromarker of human social coordination. // Proc.17tl. Acad. Sci. USA. 2007. V.104. P.8190-8195.

[8] RozenkrantsB.,Olofsson J.K., and Polich J. Affective visual event-related potentials: Arousal, valence, and repetition effects for normal and distorted pictures. // Int. J. Psychophysiol. 2008. V.67 (2).P.114-123.
[9] Babiloni C, Vecchio F, Infarinato F, Buffo P, Marzano N, Spada D, Rossi S, Bruni I,Rossini PM, Perani D. Simultaneous recording of electroencephalographic data in musicians playing in ensemble. Cortex, 2011 47(9):1082-90

[10] Babiloni C, Buffo P,Vecchio F,Marzano N, Del Percio C, Spada D, Rossi S, Bruni I,Rossini PM, Perani D. Brains "in concert": Frontal oscillatory alpha rhythms, and empathy in professional musicians. Neuroimage. 2011 13; 60(1):105-116.

[11] Pessoa L. Seeing the World in the Same Way. // Science. 2004. V.303. N.5664, P.1617 - 1618.

[12] Kay K.N., Naselaris T., Prenger R.J., Gallant J.L. Identifying natural images from human brain activity. // 17ture. 2008. V.452.P.352-355.

[13] Jang E.H.,Suk J.W., Lee Y.C., and Sohn J.H. Brain function, and EDA response while seeing fearful film clips. // Int. J. Psychophysiol. 2008. V.69 (3).P.271-272.

[14] Kreibig, S.D., Wilhelm, F.H., Roth, W.T., and Gross, J.J. Cardiovascular, electrodermal, and respiratory response patterns to fear- and sadness-inducing films. // Psychophysiology. 2007. V.44. P.787-806.

[15] Jääskeläinen IP, Koskentalo $\mathrm{K}$, Balk MH,Autti T, Kauramäki J,Pomren C, Sams M. Inter-subject synchronization of prefrontal cortex hemodynamic activity during natural viewing. Open Neuroimag J. 2008; 2:14-9.

[16] Kauppi JP,Jääskeläinen IP, Sams M, Tohka J. Inter-subject correlation of brain hemodynamic responses during watching a movie: localization in space, and frequency. Front Neuroinform. 2010 Mar 19; 4:5.

[17] A.A. Aldersons The mechanisms of electrodermal reactions, //Riga, Zinatne. 1985.

[18] Dawson M.E., Shell A.M., Filion D.N. The electrodermal system. // Principles of Psychophysiology (Ed. J.T. Cacioppo, L.G. Tassi17ry) Cambridge: Cambridge Univ. Press, 1990. P.295-324.

[19] Sokolov, E.N. Neural Mechanisms of Memory and Learning, Moscow. Nauka. 1981.

[20] Collet C., Roure R., Rada H.DittmarA., Vernet-MauryE. Relationships between performance, and skin resistance evolution involving various motor skills. // Physiol. and Behav. 1996. V.59. No.4-5. P. 953-963.

[21] Dementienko, V.V., Dorokhov, V.B., Koreneva, L.G., Markov, A.G., Tarasov, A.V., Shakhnarovich, V.M. Hypothesis on the nature of electrodermal reactions. FiziologiyaCheloveka Magazine, 2000 A. T 26. N.2, 124-131

[22] Dorokhov, V.B., Dementienko, V.V., Koreneva, L.G., Markov, A.G., Shakhnarovich, V.M. Electrodermal indicators of subjective perception of activity errors in a somnolent condition. ZhurnalVyssheiNervnoiDeyatelnosti Magazine. 2000. T.50. No.2. p.206-218.

[23] Dementienko, V.V., Dorokhov, V.B., Koreneva, L.G., Markov, A.G., Tarasov, A.V., Shakhnarovich, V.M. Sexual differences in electrodermal activity on various wakefulness levels. // FiziologiyaCheloveka Magazine. 2000 B. T.26. N.4, p.136-139. 
[24] Dementienko, V.V., Dorokhov, V.B., Markov, A.G., Mirgorodsky, V.I., Gerasimov, V.V., Peshin, S. V., Comparison of individual patterns of electrodermal reactions displayed by different subjects while viewing similar video materials. Proceedings of the Fourth International Conference on Cognitive Science. Tomsk: 2010. Vol. 1, p. 236.

[25] Zhuravlyov A.L. Psychology of joint activities. Moscow. Institute of Psychology of RAS 2005.
[26] Cacioppo J. T. Social neuroscience: Autonomic, neuroendocrine, and immune responses to stress. // Psychophysiology. 1994. V.31 (2).P.113-128.

[27] Norman G.J., Cacioppo J.T., Berntson G.G. Social neuroscience. // Wiley Interdisciplinary Reviews: Cognitive Science.2010. V.1.P.60-68.

[28] Fere C. Note sur les modifications de la resistance electrique sous l'influence des excitations sensorielleset des emotions. C. R. Soc. Biol.1888.Paris 5: 217-9. 